\title{
Toward a Complete Inclusion of the Vector Information in Morphological Computation of Texture Features for Color Images
}

\author{
Andrey Ledoux, Noël Richard, Anne-Sophie Capelle-Laizé, and \\ Christine Fernandez-Maloigne \\ Université de Poitiers, XLIM-SIC UMR CNRS 7252, Bât. SP2MI, \\ Bd Marie et Pierre Curie, Téléport 2, 86962 Futuroscope Cedex, France \\ \{firstname. surname\}@univ-poitiers.fr
}

\begin{abstract}
In this paper, we explore an original way to compute texture features for color images in a vector process. To do it, we used a dedicated approach for color mathematical morphology using distance function. We show in this paper the scientific construction of morphological spectra and preliminary results using Outex database.
\end{abstract}

Keywords: Color image, texture features, vector information.

\section{Introduction}

In this paper, we propose to compute texture features using a new mathematical morphology algorithm based on distance function. In literature several developments appear for multivalued mathematical morphology (MM) trying to extend the morphological texture analysis to vector contents [2]. However no methods take fully into account vector information of color. Non-linearity induced by this lack impacts the features accuracy.

In paper [5], we proved that mathematical morphology method based on perceptual distance fuction (called CCMM) is the most accurate for color texture characterization on color synthetic. Our objective is to assess the lack of accuracy of method which not take into account the vector information of color. In this work, we propose a first framework to compute such texture features from the CCMM method. So, we use pattern spectrum, morphological covariance and fractal signature in a classification process and show first results.

\section{Morphological Spectrum}

First, we detail pattern spectrum, morphological covariance and fractal signature algorithm. Then we describe in section 3 the ordering process to construct lowlevel morphological operators. To better understand their response to texture, we will use a simple pattern construction (fig. 1) with different pattern size (same vector contrast and inter-object distance). Caption color of figures 1a to $1 \mathrm{e}$ correspond to curve color on fig. 2 . 


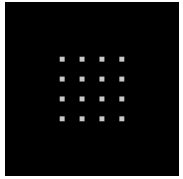

(a) Size 5

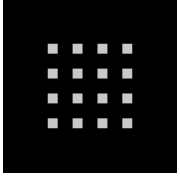

(b) Size 10

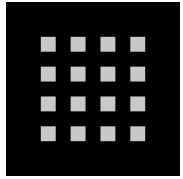

(c) Size 15

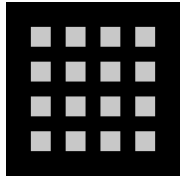

(d) Size 20

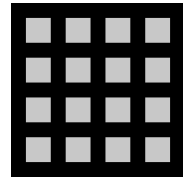

(e) Size 25

Fig. 1. Images of squares with different sizes

\subsection{Pattern Spectrum}

Pattern Spectrum or granulometry is a shape-size descriptor developed by Matheron [8]. The object description is obtained with successive morphological opening $(\gamma) / \operatorname{closing}(\varphi)$ that deletes object from smallest to largest. The pattern spectrum $P S$ (eq. 1) of image $f$ is the object distribution depending on $g$ the structuring element of size $n$ :

$$
\begin{gathered}
P S_{\mathfrak{g}}(f, g)(n)=V_{G_{\mathfrak{g}}}(f, g)(n+1)-V_{G_{\mathfrak{g}}}(f, g)(n) \\
\text { with } \quad V_{G_{\mathfrak{g}}}(f, g)=\left[G_{\mathfrak{g}}(f, n g)_{n=\infty . .1}, A G_{\mathfrak{g}}(f, n g)_{n=0 . . \infty}\right] ; \\
G_{\mathfrak{g}}(f, n g)=\frac{\operatorname{Vol}\left(\gamma_{\mathfrak{g}}(f, n g)\right)}{\operatorname{Vol}(f)} ; \quad(3) \quad A G_{\mathfrak{g}}(f, n g)=\frac{\operatorname{Vol}\left(\varphi_{\mathfrak{g}}(f, n g)\right)}{\operatorname{Vol}(f)} ; \\
\text { and } \quad \operatorname{Vol}(f)=\sum_{x}(f(x))
\end{gathered}
$$

Pattern spectrum obtained for images in figure 1, allow to identify object size (left part) and distance between objects (right part). The magnitude change of the left part is linked to the increasing number of pixel of objects.

\subsection{Morphological Covariance}

Morphological Covariance [11] characterizes the texture by analyzing the object appearance frequency in the image. Morphological covariance is calculated from a function $\xi$ and a pair of points $P_{2}$ separated by a vector $\vec{v}$ (eq. (6). The function $\xi$ commonly used is an erosion but the opening was also used [3]. The main writing form is the normalized one:

$$
K_{\mathfrak{g}}\left(f, P_{2, v}\right)=\operatorname{Vol}\left(\xi_{\mathfrak{g}}\left(f, P_{2, v}\right)\right)(6) \quad K_{\mathfrak{g}}^{n}\left(f, P_{2, v}\right)=\frac{\operatorname{Vol}\left(\xi_{\mathfrak{g}}\left(f, P_{2, v}\right)\right)}{\operatorname{Vol}(f)}
$$

The vector $\vec{v}$ can take different directions and different lengths, then $K\left(f, P_{2, v}\right)$ is the concatenation of the different response among $\vec{v}$. The morphological covariance response to images of squares (fig. 2b) shows the symmetrical texture behaviour for the horizontal and vertical axis and for the diagonal axis. The right part of the response for each $\vec{v}$ presents a peak located proportionally to the object size. 


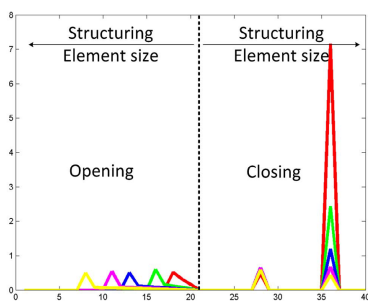

(a) Pattern spectrum

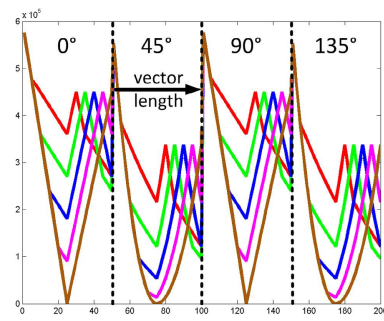

(b) Morphological covariance

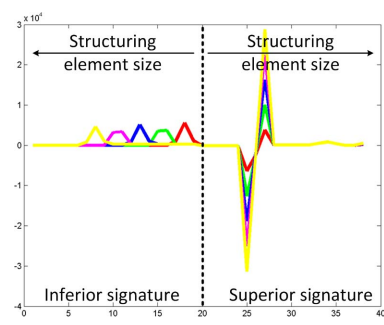

(c) Fractal signature

Fig. 2. Morphological spectra obtain for figures of squares (fig. 1a to 1e)

\subsection{Fractal Signature}

Fractal Signature expression is derived from the fractal dimension calculation introduced by Mandelbrot [7. It characterizes the complexity of the objects whose structure is invariant under scaling. This algorithm is derived from the calculation of the Minkowski-Boulingan dimension.

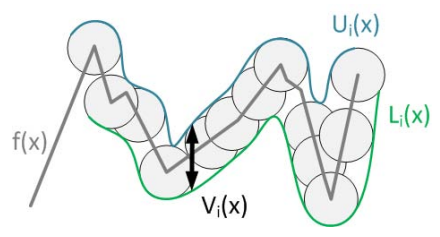

Fig. 3. Covering blanket principle

The fractal signature is obtained by evaluating the volume evolution between two surfaces enveloping the image surface at different scales $i$ (fig. 3). Both surfaces are called upper $U$ and lower $L$. The surface definition is equivalent to an erosion (eq. 12)/dilation (eq. 13) using the non-flat structuring element $g$ with diamond shape and size $3 \times 3$.

$$
\operatorname{Sign}_{i}=\operatorname{Var}_{S_{i}}-\operatorname{Var}_{S_{i-1}}
$$

$$
\begin{aligned}
& \text { with } \quad \operatorname{Var}_{S_{i}}=S_{i}-S_{i-1} ; \quad(9) \quad S_{i}=\frac{\operatorname{Vol}_{i}-\operatorname{Vol}_{i-1}}{2} ; \\
& V l_{i}=\sum_{x}\left(U_{i}(x)-L_{i}(x)\right) ; \quad(11) \quad L_{i}(x)=\varepsilon_{\mathfrak{g}}\left(f, i g_{\text {diam }}\right)(x) ; \\
& \text { and } \quad U_{i}(x)=\delta_{\mathfrak{g}}\left(f, i g_{\text {diam }}\right)(x)
\end{aligned}
$$

In this paper, we use the inferior and superior signature as described by Peleg [10]. These signatures are obtained in the same way using the inferior volume $\operatorname{Vol}_{\text {inf }}$ (eq. 14) and superior volume $\operatorname{Vol}_{\text {sup }}$ (eq. 15). 


$$
\operatorname{Vol}_{\text {inf }_{i}}=\sum_{x}\left(f(x)-L_{i}(x)\right)(14) \quad \operatorname{Vol}_{\text {sup }_{i}}=\sum_{x}\left(U_{i}(x)-f(x)\right)
$$

Computing the fractal signature for the image sequence of figure 1 produces something close to the pattern spectrum response. Object size and distance are directly obtained, and indications of the object count (ratio between size and pixel count) should be obtained for a given contrast.

\section{Mathematical Morphology Based on Distance Function}

Compute pattern spectrum, morphological covariance and fractal signature requires the definition of color erosion, dilation, opening and closing. Consequently, color value ordering must be defined. The most widely used methods to define minimum $(\bigvee)$ and maximum $(\bigwedge)$ operations in color spaces are two equivalent approaches, the lexicographic order or order based on priority expressed between color axis 64. Consequently, the dilation and erosion operators by the structuring element $g$, in n-dimensional space, can be expressed by:

$$
\delta_{\mathfrak{c}}(f, g)(x)=\bigvee_{x \in \mathcal{D}_{f}, y \in \mathcal{D}_{g}}\{f(x-y)\} \quad(16) \quad \varepsilon_{\mathfrak{c}}(f, g)(x)=\bigwedge_{x \in \mathcal{D}_{f}, y \in \mathcal{D}_{g}}\{f(x-y)\}
$$

where $\mathcal{D}_{f}$ and $\mathcal{D}_{g}$ are respectively the image support and the structuring element support.

In the majority of color mathematical morphology construction, vectorial information of color is not take into account. So we propose a new method called "Convergent Color Mathematical Morphology" (CCMM). In the proposed method, the basic order relation between two color coordinates is built according to the distance from a reference color coordinates $\left(O^{-\infty}\right.$ and $\left.O^{+\infty}\right)$. Then the minimum and the maximum between two colors, $C_{1}$ and $C_{2}$, could be:

$$
\bigwedge\left(C_{1}, C_{2}\right) \Leftrightarrow\left|\overrightarrow{C_{1} O^{-\infty}}\right| \leq\left|\overrightarrow{C_{2} O^{-\infty}}\right| ; \bigvee\left(C_{1}, C_{2}\right) \Leftrightarrow\left|\overrightarrow{C_{1} O^{+\infty}}\right| \leq\left|\overrightarrow{C_{2} O^{+\infty}}\right|
$$

where $O^{-\infty}$ and $O^{+\infty}$ are respectively the convergence points of the erosion and the dilation. In equations (18), the vector norm $|$.$| uses the perceptual dis-$ tance $\Delta E$ computed in CIELAB. In a previous work, we showed that the $\Delta E$ color distance is most accurate than other formulations or expressions in other color spaces. The (18) expression ensures the linear convergence in a perceptual sense toward the color coordinates chosen. But they don't construct a total order as required. The complete description and the validation of a total order are not the subject of this paper, so they won't be detailed here. The definition of the maximum color coordinates on the image support $\mathcal{D}_{f}$ and the structuring element support $\mathcal{D}_{g}$, for the dilation is defined by the equation 19 


$$
\begin{aligned}
\bigvee_{x \in\left(\mathcal{D}_{f} \cap \mathcal{D}_{g}\right)}\{f(x)\} & =\left\{C_{y}, C_{y}=\bigvee_{\forall C_{x} \in \mathcal{S}_{\mathcal{D} 9}}\left\{C_{x}^{\beta}\right\}\right\} \\
\text { with } \mathcal{S}_{\mathcal{D} 9} & =\left\{C_{y}: C_{y}=\bigvee_{\forall C_{x} \in \mathcal{S}_{\mathcal{D} 8}}\left\{C_{x}^{\alpha}\right\}\right\} ; \\
\mathcal{S}_{\mathcal{D} 8} & =\left\{C_{y}: \overrightarrow{\left|C_{y} O^{-\infty}\right|}=\bigvee_{\forall C_{x} \in \mathcal{S}_{\mathcal{D} 7}}\left\{\overrightarrow{\left|C_{x} O^{-\infty}\right|}\right\}\right\} ; \\
\mathcal{S}_{\mathcal{D} 7} & =\left\{C_{y}: \overrightarrow{\left|C_{y} C_{i}\right|}=\bigvee_{\forall C_{x} \in \mathcal{S}_{\mathcal{D} 6}}\left\{\mid \overrightarrow{\left|C_{x} C_{i}\right|}\right\}\right\} ; \\
\text { and } & \mathcal{S}_{\mathcal{D} 6}=\left\{C_{y}: \overrightarrow{\left|C_{y} O^{+\infty}\right|}=\underset{\forall x \in\left(\mathcal{D}_{f} \cap \mathcal{D}_{g}\right)}{\left.\left\{\left|C_{x} O^{+\infty}\right|\right\}\right\}}\right.
\end{aligned}
$$

\section{Preliminary Results}

To assess the discriminatory aspect of the various color texture attributes we use the Outex database. In this paper we focus on discrimination of texture with the same acquisition conditions. So we use the group of images "Outex_TC_00013" which contains 68 images sorted into 12 categories. Facing reduced number of images in certain categories, an image is considered as a class and is divided into thumbnail of size $128 \times 128$ pixels [1].

First, we use only three images (figure 4) and we study the spectra variability of the 20 thumbnails belonging to each image. Figures [5] to 7 are successively the superposition of the pattern spectrum, the morphological covariance and the fractal signature obtained for the 20 thumbnails of these three images.

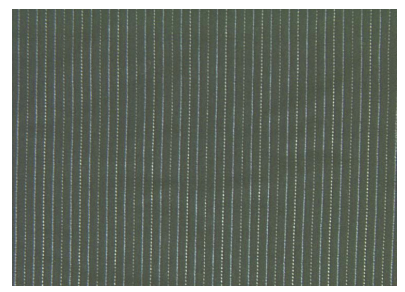

(a) canvas009

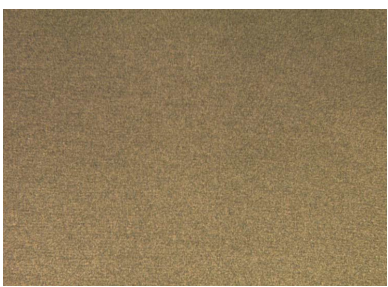

(b) sandpaper003

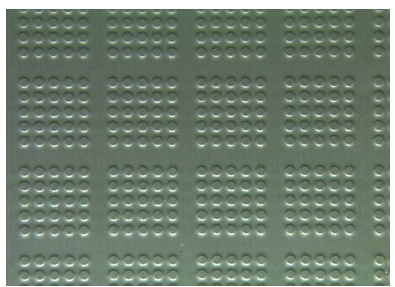

(c) carpet001

Fig. 4. Three used images of Outex database

Pattern spectrum and fractal signature have similar behaviors. These spectra have a low standard deviation which characterize a low sensitivity to clarity variations. So they are low noisy. This behavior is explained by a treatment based on contrasts rather than on absolute values. In addition, these spectra have significant differences according to textures variations. Figures $5 \mathrm{a}$ and $5 \mathrm{c}$ 


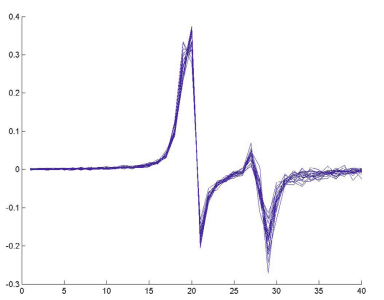

(a) Pattern spectrum

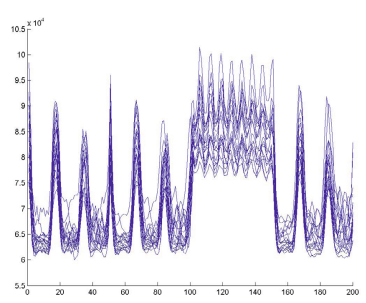

(b) Covariance

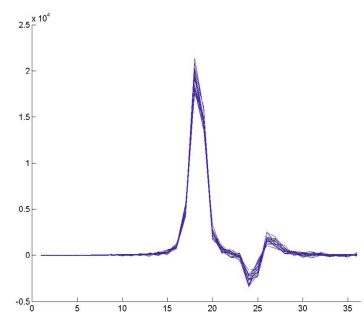

(c) Fractal signature

Fig. 5. Superposition of 20 spectra of thumbnails for image canvas009-inca-100dpi-00 of Outex database for different methods

are pattern spectrum and fractal signature examples. These figures show spectra with a peak characterizing the distance between vertical lines (around 20 pixels). In the same way, figures $7 \mathrm{a}$ and $7 \mathrm{c}$ highlight the circle frequency (around 30 pixels). High frequencies of figure $4 \mathrm{~b}$ are characterized by a dominant peak for very low values (fig. 6a and 6c).

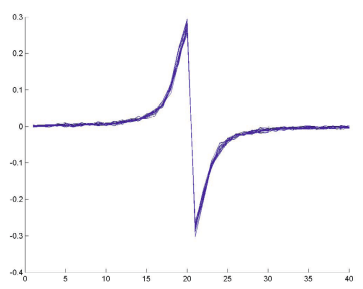

(a) Pattern spectrum

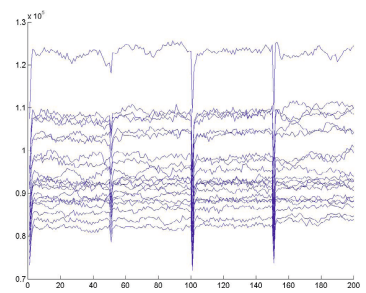

(b) Covariance

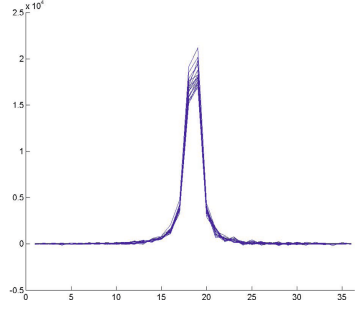

(c) Fractal signature

Fig. 6. Superposition of 20 spectra of thumbnails for image sandpaper003-inca-100dpi00 of Outex database for different methods

Morphological covariance has a strong ability to caracterize repetition of patterns in a texture. Figure $5 \mathrm{~b}$ is an example of spectra characterizing pattern repetition. Vertical lines are characterized with a peak at low frequency for angles of $0^{\circ}, 45^{\circ}$ and $135^{\circ}$. Angle of $90^{\circ}$ highlights canvas fibers on the vertical line. In the case of figure $4 \mathrm{c}$, patterns are larger than those present in the canvas images (fig. 4a), then covariance spectra are composed of a single peak for the four directions. For images of fine texture random (fig. 4b) the covariance presents low variations. Finally, morphological covariance spectra have the disadvantage that have an important standard deviation due to the sensitivity to illumination change. This problem increases with the saturation of images (fig [6b). 


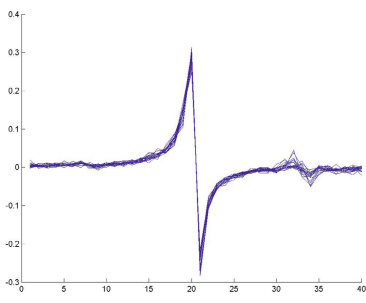

(a) Pattern spectrum

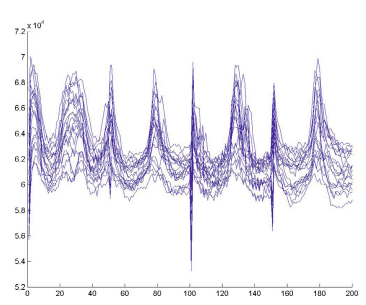

(b) Covariance

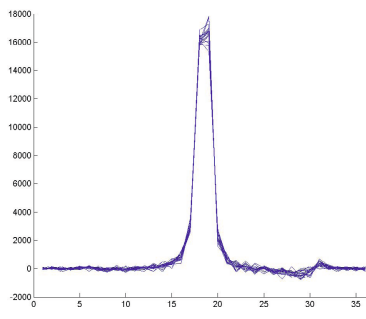

(c) Fractal signature

Fig. 7. Superposition of 20 spectra of thumbnails for image carpet001-inca-100dpi-00 of Outex database for different methods

In a second step, we use all the group of images "Outex_TC_00013" to compare the different spectra inside a texture classification process. The classification is not performed to evaluate the performance of classification tools but to evaluate the vectorial texture spectra. So we use the classification algorithm proposed by Arvis [1] based on k-nearest neighbors. Table 1] contains results using one or three neighbors. Spectra of vector texture take into account a part of color information but this information is analyzed as a color contrast. So in third column, color average is added to texture features for classification. Differentiation of two similar textures with different colors is then possible.

This classification highlights the importance of color information in methods. Results obtained with the spectra of vector texture are worse than using greyscale features 91. These results are due to weak spatio-chromatic complexity of textures in Outex database. Same behaviour is obtained for others image database, nevertheless more complex images as fractal images justify such vector texture features.

Table 1. Correct classification percentage for different color texture features with the KNN method using 1 or 3 neighbors; using or not the color average

\begin{tabular}{|l|c|c|c|}
\hline Methods & KNN $(\mathrm{k}=1)$ & KNN $(\mathrm{k}=3)$ & KNN $(\mathrm{k}=1)+$ color average \\
\hline Pattern spectrum & 72.35 & 73.08 & 83.38 \\
Covariance & 33.50 & 36.02 & 43.23 \\
Fractal signature & 49.55 & 50.58 & 53.08 \\
\hline
\end{tabular}

\section{Conclusion}

In this paper and for the first time, we proposed morphological spectra correct among the perception, using a perceptual distance function. This construction is fully generic and can be extended to spectral mathematical morphology and spectral texture features.

We shown the interest and readability of these spectra for basic textures, and we investigated their discriminating aspect in a preliminary classification task. 
As the existing texture image database present a low level of spatio-chromatic complexity, classical grey-level approaches are more efficient than vector ones. First try on images with highest spatio-chromatic complexity, as fractal images, shown that under this condition vector features becomes more discriminant than grey-level or marginal approaches.

For the moment, no texture image database is really adapted for colour texture classification, due to a reduced spatio-chromatic complexity. So it is not possible to assess performances between vector texture features, and/or the others sets of texture features. An international work is in progress to specify and construct such image database for colour and spectral data.

\section{References}

1. Arvis, V., Debain, C., Berducat, M., Benassi, A.: Generalization of the cooccurrence matrix for colour images: application to colour texture classification. Image Analysis and Stereology 23(1), 63-72 (2004)

2. Angulo-Lopez, J., Serra, J.: Modelling and segmentation of colour images in polar representations. Image and Vision Computing 25(4), 475-495 (2007)

3. Aptoula, E.: Comparative study of moment based parameterization for morphological texture description. Journal of Visual Communication and Image Representation (2012)

4. Hanbury, A., Serra, J.: Morphological operators on the unit circle. IEEE Transactions on Image Processing 10(12), 1842-1850 (2001)

5. Ledoux, A., Richard, N., Capelle-Laizé, A.S., et al.: The fractal estimator: A validation criterion for the colour mathematical morphology. In: 6th European Conference on Colour in Graphics, Imaging, and Vision, pp. 206-210 (2012)

6. Louverdis, G., Vardavoulia, M.I., Andreadis, I., et al.: A new approach to morphological color image processing. Pattern Recognition 35(8), 1733-1741 (2002)

7. Mandelbrot, B.B.: Fractals: Form, Chance, and Dimension. W.H. Freeman and Company (1977)

8. Matheron, G.: Random sets and integral geometry. John Wiley \& Sons Inc., New York (1975)

9. Ojala, T., Pietikinen, M., Menp, T.: Multiresolution gray-scale and rotation invariant texture classification with local binary patterns. IEEE Transactions on Pattern Analysis and Machine Intelligence 24(7), 971-987 (2002)

10. Peleg, S., Naor, J., Hartley, R., et al.: Multiple résolution texture analysis and classification. IEEE PAMI 6, 518-523 (1984)

11. Serra, J.: Image Analysis and Mathematical Morphology, vol. I. Academic Press (1982) 